\title{
Optimizing motor-timing decision via adaptive risk-return control
}

Qirui $\mathrm{Yao}^{1^{*}}$, Yutaka Sakaguchi $^{1}$

1 Department of Mechanical Engineering and Intelligent Systems, Graduate School of Informatics and Engineering, University of Electro-Communications, Chofu, Tokyo, Japan

* qyao2017@uec.ac.jp

Email: yutaka.sakaguchi@uec.ac.jp (Yutaka Sakaguchi)

\begin{abstract}
Human's ability of optimal motor-timing decision remains debated. The optimality seems context-dependent as the sub-optimality was often observed for tasks with different gain/loss configurations: people achieved optimality with symmetric gain configuration but not with asymmetric configuration. In the current study, we designed a temporal decision-making task where participants could adjust the sensitivity parameter (i.e., risk-return trade-off) of the gain function, in order to testify whether people could optimize the responses for asymmetric gain configuration by adjusting the sensitivity parameter. Participants were asked to click a point within a certain spatial region at a specified timing, where the click timing determined the reward whilst the click position determined the sensitivity parameter. We prepared three types of gain functions (symmetric, risk-after and risk-before conditions) and tested whether or not the participants achieved Bayesian optimality irrespective of gain structure. Most
\end{abstract}


participants' performance reached optimality not only in the symmetric condition but also in the asymmetric condition, albeit some discrepancies from optimality observed in the risk-before condition. This confirmed that people could achieve Bayesian optimality even for asymmetric gain configuration. We argued that the adaptive risk-return is beneficial for the performance optimality.

Keywords: Bayesian decision model, Decision making, Motor timing

\section{Introduction}

Humans always have to decide when to execute a motor action in daily life situations. Each action corresponds to a specific gain/loss. We often find ourselves in a dilemmatic scenario for action selection. Imagine that you, standing on a road-side, detected a mad dog charging towards you, and faced an impromptu decision about when to get away by crossing the busy road. Sooner reaction would increase the chance of dodging the attack but have less time to examine the traffic condition. Taking time to find a safer side to cross the road, on the other hand, might not survive the dog attack. Among the infinite choices, a particular time point at which to execute the action that could minimize the loss and maximize the gain might exist.

The mathematical modeling using Bayesian decision-making theory (BDT) allows us to find the optimal solution for the temporal decision-making scenario, such as the one mentioned above [1,2]. It has been documented that human participants' performance in various motor-timing tasks supports the BDT [3 5]. In line with this theory, any temporal decision-making scenarios can be modeled as follows.

$$
E[G]=\int G(t) p\left(t \mid t_{e}, \sigma_{t}^{2}\right) d t
$$

$G(t)$ is the gain function mapping from all possible timing of motor executions to their

corresponding outcomes. The $p\left(t \mid t_{e}, \sigma_{t}^{2}\right)$ defines the probability distribution of the 
temporal input $t$ incurred from choosing a timing strategy $t_{e}$, given a timing response variance $\sigma_{t}^{2}$. Note that mathematically the Bayesian optimality is achieved by maximizing the expected gain function Equation (1). In the temporal decision-making scenarios to achieve optimal performance, participants were required to find a trade-off between the timing variance of motor action and the risk-return configuration of the gain fucntion 6 8. The risk-return trade-off predicted that there existed a positive relationship between the motor-timing and the corresponding response variance 8]. Therefore, participants with greater timing variance would prefer low-risk and low-return, and conversely for the participants with smaller timing variance.

According to the previous literature 9 15, whether humans could achieve optimal performance in various tasks remained questioned. In particular, recent studies regarding the coincident timing tasks suggested that the motor-timing was not universally optimal but depended on the risk-return configurations 12,13 . Ota et al. 12 discovered that participants tended to exhibit risk-seeking behaviors when the risk-return configuration (i.e., gain function of timing responses) was asymmetric whereas risk-neutral (i.e., optimal) performance was observed with symmetric gain functions. Note that in both conditions all participants experienced identically-structured gain functions, irrespective of their motor timing variability. However, in another research in which experimenters adjusted the gain/loss ratio for each participant according to his/her estimated timing variance, participants were able to determine the motor-timing optimally even with asymmetrical gain configurations [16]. These contrasting findings indicated the structure of the gain function might not be a decisive factor of the performance optimality.

Regarding the risk-return control, Nagengast et al. [8, 17] argued that humans did not only consider the mean payoffs but were also sensitive to the payoff variance. In their experiment involving speed-accuracy trade-off, participants were allowed to control 
the variance of the gain function by choosing the movement speed. By equalizing the optimal speed, the risk sensitivity (i.e., risk-neutral, risk-aversive and risk-seeking) for each participant could be assessed by varying the payoff variance. In contrast to the previous studies using pre-experimentally fixed gain structure, Nagenagst 's design firstly introduced an adjustable risk-return that constructed a mapping between the response variance and the payoff variance [8]. Focusing on the motor-timing tasks, we assumed that implementing the adjustable risk-return control in a trial-by-trial fashion would lead to optimal performance.

Here in the present study, we designed a temporal decision-making task to investigate the strategies with three types of gain functions (symmetric, risk-after and risk-before conditions). In our study, we designed an adaptive control to allow participants adjust the steepness of the gain function in a trial-by-trial fashion. If the sub-optimality discovered with asymmetric gain functions was a result of the given structure of the gain function, the sub-optimality could be altered under the adaptive control. Each participant's performance was evaluated by a Bayesian decision-making model incorporating both timing and spatial variance. In contrast to the previous studies 12 13], we found participants not only reached optimal performance in the symmetric condition but also in the risk-after condition, although some retained sub-optimal in the risk-before condition. Our research provides experimental evidence regarding the effectiveness of adaptive risk-return control.

\section{Method}

\section{Participants}

Twenty-one right-handed participants (five females) took part in the symmetric condition. Eleven of them (two females) took part in the risk-after condition, and the 
remaining ten (three females) participants took part in the risk-before condition. All participants reported having normal visual and hearing acuity. All completed the whole experimental session, and each received a reciprocal reward for participation (a prepaid book voucher of 1,000 JPY, about 9 USD) was given for every experimental hour). This experiment was approved by the University of Electro-Communications Institutional Review Board for Human Subjects Research (\#14007), and was in accordance with the ethical standards in the Declaration of Helsinki. We obtained written informed consent from all participants.

\section{Apparatus}

The experiment was conducted in a quiet lab room under fluorescent light. Participants sat at a distance of approximately $56 \mathrm{~cm}$ from the display. Stimuli were displayed over a white background on a 23-inch LCD screen (LG Inc.) with a $1920 \times 1200$ resolution and a refresh rate of $100 \mathrm{~Hz}$. The signal tones $(1000 \mathrm{~Hz})$ and the clicking tone $(900 \mathrm{~Hz})$ used in the experiment session were played through a headphone. An optical mouse with a scroll wheel (Dell Inc.) was used for the clicking performance. Experimental stimuli were generated and controlled using Inquisit 5.0 [18.

\section{Stimuli and Experimental Procedure}

In each experimental condition, the target stimulus (radius: 500 pixels) was a semi-circular ring-shaped and grey object (Figure 19). The target stimulus was equally differentiated into eighteen equal-area parts by black lines. A fixation circle (FC) was presented at the center of the ring, and all points on the stimulus were equidistant from the FC. All operations were executed through mouse click. Participants clicked the FC to initiate each trial (Figure 1b). After a random interval (0.5 to $1 \mathrm{~s})$, the target interval was demarcated by two signal tones. The target interval was fixed at $2.3 \mathrm{~s}$ for all trials, 
which was adopted from Ota et al. to make direct comparisons 12. Participants were asked to initiate the mouse cursor from the FC to the target region after the presentation of the second tone and clicked at a certain timing. Mouse click was recorded as valid response. As response feedback, the clicking tone $(900 \mathrm{~Hz})$ presented for $0.05 \mathrm{~s}$ and visual feedback (red spot, diameter 10 pixels) indicating the clicked position remained on the display until the onset of the next trial. The target time was also provided by a tone $(1000 \mathrm{~Hz})$ for reference. Providing the auditory feedback ensured participants could attend to the temporal location of the response relative to the target tone. Prior to the experiment, each participant received several practice trials adequate to warm up their motor-timing responses. Each experimental condition comprised five blocks with each containing 30 trials. Participants were asked to search for movement direction and response time to maximize the reward scores received throughout 150 trials $(5$ blocks $\times 30$ trials). Block 1 and 2 served as learning sessions in which participants were allowed to explore without any restrictions as well as familiarize themselves with the task performance and the structure of the gain function. From Block 3 to 5 , the search zone was constricted (its border was signaled by four blue dots, 10 pixels) in subtractive steps $(90,40,20$, in degree). The center of the search zone was determined by the average movement direction in the previous block. For example, if the averaged movement direction in Block 2 was 30 deg, the search zone in Block 3 was $30 \pm 90$ deg. Once the search zone appeared, participants were asked to select movement direction within the search zone. This design aimed to avoid unconverged exploring patterns after learning sessions and to maintain response variance.

The number of scores received on each trial and the block-averaged score were shown immediately after the response was released. There were three experimental conditions assigned with different score structures. All participants first performed the risk-symmetric condition (Figure 2a) in which a modified Gaussian gain function was 
Fig 1. Stimulus and Task. (a) Experimental stimulus. The stimulus consists of a semi-circular ring and a central fixation circle. The movement direction subtended by the directional angle $r$ is delimited by the dash line (about $168 \mathrm{deg}$ ) hidden from view. The click position is marked by a red spot. (b) Each trial was initiated by clicking the fixation circle. Participants were asked to generate the timing response bringing the largest reward. This was achieved either by reproducing the target interval (in symmetric condition) or producing a timing a little bit longer/shorter than the target interval (in risk-before/risk-after conditions). The reward points and the click tone were fed back.

adopted. Upon completion, participants were assigned to the risk-asymmetric conditions (risk-before Figure 2b or risk-after Figure 2r). The shape of these gain functions was instructed using oral and visual aids prior to the experiment.

Fig 2. Shapes of gain function in three experimental conditions. (a) Symmetric condition. The reward points decrease with respect to the symmetry axis. (b) Risk-before condition. Negative points are imposed if the responses are made before the target time. (c) Risk-after condition. The structure is a mirror-reflection of the risk-before structure. In all conditions, participants could control the shape of the gain function by choosing the directional angle of movement. Each panel displays two curves that demonstrate the two extremes of the gain functions.

\section{Bayesian modeling of optimal decision-making}

We designed a Bayesian decision-making model that consists of five elements: the planned action (click) timing $t_{e}$, the actual action timing $t$, the planned movement direction $r_{e}$, the actual movement direction $r$ (defined by the directional angle of the 
clicked position), and the reward function $G(t ; r)$ associated with the action. Follow the previous model [12], the actual motor timing $t$ is assumed to be distributed according to a Gaussian distribution due to the internal timing noise $\sigma_{t}$.

$$
p\left(t \mid t_{e} ; \sigma_{t}\right)=\frac{1}{\sqrt{2 \pi \sigma_{t}^{2}}} \exp \left[-\frac{\left(t-t_{e}\right)^{2}}{2 \sigma_{t}^{2}}\right]
$$

The actual movement direction was modeled by a Gaussian distribution $\left(r_{e}, \sigma_{r}\right)$ on the assumption that the selected directions could converge to a particular end-point after restricting the search zone, where $\sigma_{r}$ is the standard deviation of directional motor noise. The number of reward received on each executed motor action was specified by the gain functions.

In the risk-symmetric condition, the gain was determined by a modified Gaussian function.

$$
G(t ; r)=\frac{10}{\sigma_{(r)}^{0.7}} \exp \left[-\frac{(t-T)^{2}}{2 \sigma_{(r)}^{2}}\right]
$$

The maximum points were given where the executed timing $t$ was equal to the target interval $T$ ( $t=T$, i.e., coincident timing), and received points reduced symmetrically with increased timing error from $T$ given the $r$ was fixed (Figure 2 a).

In the risk-before condition, the gain function contained a "basin" and a "cliff " structure where any click actions occurred before the target time was equally penalized and that occurred after the target time brought the points reduced identically to the defined Gaussian structure (Figure $2 \mathrm{p}$ ).

$$
G(t ; r)=\left\{\begin{array}{r}
\frac{10}{\sigma_{s}^{0.7}} \exp \left[-\frac{(t-T)^{2}}{2 \sigma_{s}^{2}}\right], t \geqslant T \\
-5, t<T
\end{array}\right.
$$

On the contrary, the risk-after condition imposed the negative gain after the target time, encouraging earlier responses. The gain structure was a mirror image of Equation (6) with respect to the target time (Figure 2k).

In all three conditions, the sensitivity of $t$ to the point depended on the sensitivity 
parameter $\sigma_{s}$ of gain function, which, formed a linear relationship with the movement direction $r$.

$$
\sigma_{s}=\left\{\begin{array}{c}
\sigma_{\min ,}, r<r_{\min } \\
a\left(r-r_{\min }\right)+\sigma_{\min ,}, r_{\min } \leq r \leq r_{\max } \\
\sigma_{\max ,}, r>r_{\max }
\end{array}\right.
$$

Where $a=\frac{\sigma_{\max }-\sigma_{\min }}{r_{\max }-r_{\min }}, r_{\min }=6 \mathrm{deg}, r_{\max }=174 \mathrm{deg}, \sigma_{\min }=0.023 \mathrm{~s}, \sigma_{\max }=1.15$ s. The values $\left(\sigma_{\min }, \sigma_{\max }\right)$ were used to cover the potential range of timing response variance of human participants.

Therefore, movement direction $r$ defines the risk-return control of this task, that is, a larger value of $r$ (moving leftward) brings low-risk and low-return whilst a smaller value of $r$ (mover rightward) brings high-risk and high-return, and the participant could determine the risk-return configuration by adjusting the movement direction trial-by-trial. We termed this method as adaptive risk-return control.

Taken together, an optimal decision-maker is to choose a movement direction and a response time $\left(r_{e}, t_{e}\right)$ to maximize the following expected gain function:

$$
E G\left(t_{e} ; r_{e}\right)=\int_{r_{\min }}^{r_{\max }} p\left(r \mid r_{e}\right) \int_{0}^{\infty} G(t ; r) p\left(t \mid t_{e}\right) d t d r
$$

The timing variance of each participant was estimated using the dataset excluding the first 10 trials to avoid the effect of warming-up. We used the data in Block 5 where the response variance was limited within 20 degrees to estimate the motor variance. The reason for choosing the last block to estimate the variance is to avoid the fluctuation of selecting movement directions during exploration and to stabilize the estimation. We used the globalserach function with an SQP algorithm in MATLAB [19] to calculate the optimal values $\left(t_{\text {opt }}, r_{\text {opt }}, p_{\text {opt }}\right)$ for each participant in each condition. 


\section{Data analysis}

One participant's dataset was removed from the analysis because the average timing response was negatively biased 2.5 SD from the group mean in the symmetric condition, indicating the participant did not pay attention to the task. Temporal outlier was defined as a timing response less than Q1 (the first quartile) $-1.5 \times$ IQR (inter-quartile range) or more than Q3 (the third quartile) $+1.5 \times$ IQR of its corresponding response distribution 20]. Spatial outliers were defined as the movement direction that exceeded $2.5 \pm \mathrm{SD}$ from the restricted search zone in the last block. The removed data took up $0.77 \%(23 / 3000)$ of the dataset in risk-symmetric condition, $1.27 \%(19 / 1500)$ in risk-before condition and $0.67 \%(10 / 1500)$ in risk-after condition.

The statistical models were constructed using Rstan 21] and lme4 22] packages. The basic setting for running the MCMC (Markov chain Monte Carlo) sampling was four chains (each chain contains 1500 warm-up steps and 3000 iterations). Rhat (a scale reduction factor) was used to examine the convergence of split chains.

\section{Results}

\section{Evaluation of performance of individual participants}

We computed the optimal point $p_{\text {opt }}$, the optimal timing response $t_{\text {opt }}$ and the optimal movement direction $r_{o p t}$ for each participant in each condition by maximizing Equation (8). To visualize the change in individual participant performance across blocks, we plotted the expected gains of each participant (plots of representative participants were shown in Figure 3 for the results of the other participants, see Supplementary Figures).

To analyze the individual optimality, we adopted the Relative Optimality (RO) [6], 
Fig 3. Expected gains and actual responses of representative participants in three conditions. Different levels of expected gains are depicted by contour lines. The density of contour reflects response variances (i.e., $\sigma_{t}, \sigma_{r}$ ) of each participant. Each dot represents an actual response (Block 1-5: light grey to black) of the participants. (a) Symmetric condition. (2) Risk-before condition. (3) Risk-after condition.

that is, the observed mean point $p_{\text {obs }}$ divided by the corresponding optimal point $p_{\text {opt }}$ :

$$
R O=\frac{p_{o b s}}{p_{o p t}}
$$

The RO was calculated for each participant in each condition using the post-learning dataset (i.e., block 3-5). We notice that a well-accepted criterion of "optimality" has not been given in the previous studies. In line with Dean et al. [6] and to avoid bias, we defined optimality by whether the "RO = 1" fell within the $99 \%$ confidence interval. We therefore regarded the $99 \% \mathrm{CI}$ as the optimality interval (OI). The OI was estimated by applying MCMC (Markov chain Monte Carlo) sampling method via rstan 21] in R (See Model Availability for model structure). We used non-informative priors. All running chains were successfully converged $($ Rhat $=1)$. In addition, we regarded the "RO $=0.9 "$ as a reference level for monitoring performance efficiency, as used in [6].

Figure 4 shows the Relative Optimality with respect to the estimated optimality interval (OI). Seventeen of twenty participants achieved optimality (Symmetric condition), where their corresponding ROs remained no difference from the optimal level $(\mathrm{RO}=1)$. Similar pattern was observed in risk-after condition where all participants' ROs $(\mathrm{RO}=1)$ lay within the OI. These findings were contracted to the previous study 12,13 where participants did not reach optimal performance in asymmetric conditions. On the other hand, our results supported that humans can achieve optimality in asymmetric conditions through the adaptive risk-return control. However, we observed an inconsistent performance pattern in risk-before condition, 
where three of participants (one at marginal edge of the cut-off level) were classified as optimal performers whereas the rest seven were classified as sub-optimal performers (according to the criterion of optimality). Although the sub-optimal performers existed, five of them reached the $90 \%(\mathrm{RO}=0.9)$ optimality, indicating a high level of performance efficiency $[6]$. The observed inconsistency of performance existed between two risk-asymmetric conditions indicated that the risk-direction might have an influence on the performance optimality, which will be discussed later.

Fig 4. Individual Relative Optimality in all three conditions. Relative Optimality is defined as the observed mean point devided by the optimal point. The dashed line represents the level for "optimality". The upper-limit and lower-limit of optimality interval (OI) are depicted by error bars. We computed the OI using MCMC sampling method. (a) Symmetric condition. (2) Risk-before condition. (3) Risk-after condition.

\section{Risk sensitivity across risk-conditions}

We observed that some participants' performance did not reach optimality in risk-before condition whereas participants in the risk-after condition could achieve optimal performance, even though an asymmetric gain structure was used. To further understand the observed inconsistency in task performance, we employed the concept of risk-sensitivity. In the previous studies, risk-sensivity was often employed to determine whether the chosen strategy is risk-aversive or risk-seeking $8,12,13$. The risk-sensitivity is assumed to occur when an asymmetric gain structure is implemented whereas no risk-sensitivity (a.k.a, risk-neutral) occurs in the symmetric gain.

Here, we investigated how the risk-sensitivity exhibited in the risk-assymetric conditions. To this end, we focused on the relationship between the mean observed timing response $t_{o b s}$ and the optimal timing response $t_{\text {opt }}\left(\triangle=t_{o b s}-t_{o p t}\right)$. Focusing 
on their relative values, the timing strategy was considered as risk-seeking if $t_{o b s}>t_{o p t}$ in the risk-after condition and if $t_{o b s}<t_{\text {opt }}$ in the risk-before condition, and conversely for a risk-aversive strategy. If no difference detected, the strategy was risk-neutral (i.e., optimal, timing difference equals to 0 ). We evaluated participant-averaged values of $\triangle$ in risk-after and risk-before conditions respectively, using the dataset (each condition contains 10 observations) after the learning session (block 3-5).

The mean, 95\% confidence interval (CI), and effect size Cohen's $d$ related to the group data were estimated using MCMC with non-informative priors (See Model Availability for model structure). All chains were successfully converged (Rhat $=1)$. The statistical significance was determined by whether "0" lays within the $95 \% \mathrm{CI}$. The results were plotted in Figure 5 . We found that the $\triangle$ was not different from 0 in risk-after condition $($ mean $=-0.0043, \mathrm{CI}=[-0.0394,0.0311], d=-0.0885)$. We also observed a marginal effect of $\triangle$ in risk-before condition $($ mean $=0.0423, \mathrm{CI}=[-0.0128$, 0.0969], $d=0.5509)$. These results indicate that participants remained risk-neutral in risk-after condition whereas participants tended to be risk-aversive (i.e., timed longer than optimal) in risk-before condition. Statistical analysis on the $\triangle$ between two conditions confirmed the interpretation that participants might involve in sub-optimal and risk-aversive timing strategies (mean difference $=0.0472, \mathrm{CI}=[-0.0192,0.1120], d$ $=0.6978)$.

Fig 5. Participant-averaged risk sensitivity in risk-asymmetric conditions. The error bars display the standard error. Each dot represents the averaged timing difference for each participant. 


\section{Compensation for risk-return trade-offs}

In the present experiment, movement direction $r$ controlled the sensitivity of the gain function (i.e., payoff variance). Bayesian optimal decision-making theory predicted a risk-return trade-off regarding the movement direction: individuals with high timing accuracy would prefer a small value in movement direction (high-risk high-return); otherwise, individuals would end up accepting a large value (low-risk low return) if the corresponding timing responses remained unstable [8,23,24]. Therefore, if one performed in a manner of maximizing the expected gain (i.e., to achieve Bayesian optimality), the timing variance would be positively correlated with the movement direction. We first normalized the block-averaged movement direction and the by-block timing variance for each participant respectively using z-score. In our design, "block" was a within-subject effect whereas "participant" was a between-subject effect.

Therefore, applying a simple linear regression was not appropriate in our analysis 25 . Instead, we then constructed linear mixed model using lme4 package 22 to investigate whether or not timing variance is a significant predictor of movement direction. "Block" and "participant" were considered as random effects. We compared the goodness of fitting and chose the random intercept model as the target model (for model comparison see Supplementary Materials):

Random intercept model (RIM)

$$
N M D \sim N T V+(1 \mid \text { participant })+(1 \mid \text { block })
$$

Where NMD is normalized movement direction and NTV is normalized timing variance, the model equation is written using pseudocode retrieved from 22 .

We focused on estimating the coefficient of regression and its $95 \%$ confidence interval (CI) respectively for each condition using bootstrap method. The results were shown in Table 1. We identified a significant fixed effect in risk-after condition, $t(49.98)=2.662$, $p=.0104$ and symmetric condition, $t(55.73)=6.076, p<.001$, indicating the existence 
of Bayesian risk-return trade-off. However, the fixed effect in risk-before condition was not significant $t(19.88)=-1.049, p=0.307$. The results indicated that the participants in risk-before condition might use different strategies rather than trading-off risk and returns.

Fig 6. Risk-return trade-offs in three conditions. Normalized timing variance is plotted against normalized movement direction for every individual participants across five blocks (differentiated by colored lines). (a) Symmetric condition. (2) Risk-before condition. (3) Risk-after condition.

\section{Discussion}

In the current research, we investigated whether participants could achieve optimal performance with the gain functions of modified symmetry when the risk-return trade-off were adjustable. To this end, we designed a hierarchical decision-making task in which participants chose a timing end-point to maximize the expected points. An adjustable risk-return control was implemented that allowed participants to select the shape of the gain function to match with the corresponding response variance. Using the Bayesian decision-making model, we found that participants were able to reach the optimality in the symmetric condition and the risk-after condition. In contrast, three out of ten participants ' performance in the risk-before condition was clearly sub-optimal and overal participants tended to adopt a risk-aversive strategy. Taken together, our findings concluded that optimality depended not directly on the symmetry of the gain structure but on the risk-neutral strategy induced by the adjustable risk-return control. Besides, risk-direction of temporal target (the tasks requiring the response after/before the target) might exert influence on the performance optimality.

Sub-optimality in motor timing was often observed in the decision-making tasks 
Table 1. Results of the random intercept model showing the effect of timing variance on movement direction in three conditions

\begin{tabular}{|c|c|c|c|c|c|c|c|}
\hline Factor & Variance & $S D$ & $\beta$ & $S E$ & $t$ & $p$ & $\mathrm{CI}$ \\
\hline \multicolumn{8}{|l|}{ Symmetric } \\
\hline \multicolumn{8}{|c|}{ Random factors } \\
\hline Participant & 0.3236 & 0.5688 & & & & & \\
\hline Block & 0.1926 & 0.4389 & & & & & \\
\hline Residual & 0.3491 & 0.5908 & & & & & \\
\hline \multicolumn{8}{|c|}{ Fixed factors } \\
\hline (Intercept) & & & -0.0068 & 0.2417 & -0.028 & 0.978 & $(-0.4636,0.4564)$ \\
\hline NTV & & & 0.4066 & 0.067 & 6.076 & $<.001$ & $(0.2753,0.5317)$ \\
\hline \multicolumn{8}{|c|}{ Risk-before } \\
\hline Participant & 0.1376 & 0.3710 & & & & & \\
\hline Block & 0.2388 & 0.4886 & & & & & \\
\hline Residual & 0.5979 & 0.7732 & & & & & \\
\hline (Intercept) & & & $<-.001$ & 0.2711 & 0.000 & 1.000 & $(-0.6134,0.5284)$ \\
\hline NTV & & & -0.1546 & 0.1473 & -1.049 & 0.307 & $(-0.4722,0.1268)$ \\
\hline \multicolumn{8}{|l|}{ Risk-after } \\
\hline Participant & 0.5037 & 0.7097 & & & & & \\
\hline Block & 0.0000 & 0.0000 & & & & & \\
\hline Residual & 0.2567 & 0.5067 & & & & & \\
\hline (Intercept) & & & $<.001$ & 0.2356 & 0.000 & 1.000 & $(-0.4248,0.4431)$ \\
\hline NTV & & & 0.3355 & 0.1260 & 2.662 & 0.0104 & $(0.0741,0.5654)$ \\
\hline
\end{tabular}

Note: $\mathrm{NTV}=$ normalized timing variance; the $95 \%$ confidence interval was estimated using bootstrap method 
where an asymmetric gain function was adopted $12,14,26,27$. For example, the "cliff" paradigm 12 26 in which the highest risk-return was located in the "cliff-edge" of the gain function, was recently often used as an experimental design in motor-timing tasks. Under the "cliff" paradigm, Ota et al. 12 asked participants to search for an optimal response timing to maximize the expected gains. They found that participants failed to reach the optimal performance no matter whether the penalty region was before (risk-before) or after (risk-after) the target time. By contraries, we discovered optimal performance under the risk-after condition even though adopting the "cliff" paradigm.

Under "cliff" paradigm, how did participants reach optimal performance with asymmetric gains? For a primary reason, we designed a motor task that allowed participants to adjust the shape of gain function in a trial-by-trial manner. Similar risk-return control was introduced by Nagengast et al. concerning speed-accuracy trade-off [8]. In contrast to Ota et al. [12, the adjustable risk-return control ensured that the structure of gain function mapped with the participants' corresponding timing variance, so that the risk-return trade-off could be achieved by finding out "the more appropriate" gain function from infinite ones. Participants in our tasks could soon notice the drastic change in received points if there were any mismatches occurred. Note that previous studies used pre-experimentally estimated response variance to construct gain functions. For example, in Wu's study [28], the estimated motor variance for each participant was used to configure the "motor lottery" (gain function) in the spatial target. Researchers found that participants tended to favor the riskier lottery than the safer one. Nagengast et al. 17] used the pointing variance estimated from training session to construct the target region specified by the participant-dependent hitting probability. As a result, most participants ended up underestimating their response variance (i.e., believing the response variance was smaller than the actual one). The above-mentioned two findings indicated that the distortion of response variance could 
occur if participants were not allowed to control the variance of gain function while performing the risk-return task. On the other hand, our findings suggested that providing more flexibility in risk-return control was crucial for appximating optimality.

Many motor decision-making tasks, used in the previous studies 29 30, require the knowledge of motor error to achieve optimal motor planning because perturbation often occurred from different sources. Similar in timing tasks, responses may be highly volatile especially when producing supra-second time intervals, due to the scalar property in interval estimation 31 and the involvement of cognitive time processing [32. To reduce the multiplicative errors incurred from estimating longer intervals, we provided participants with feedback about their timing errors by signaling both the response time and the target time (i.e., $2.3 \mathrm{~s}$ ). Participants could refer to the timing error to maintain timing accuracy without taking excessive trials, as we observed that most participants reached the optimal performance after five blocks (150 trials) of experiments.

Particularly in the risk-asymmetric conditions where the optimal time value required exploration, providing repeated corrective feedback could enhance the learning of internal representation of timing errors [33], and therefore led to optimal performance.

Thirdly, we introduced the penalty region to the gain function's structure, following the previous designs regarding the motor planning [4,5, 16, 23, 24, 27]. Focusing on the temporal task, Hudson et al. 16] punished early/late timing responses and discovered that participants behaved in a manner of choosing the movement times that maximized the expected rewards. Studies also found the neural basis that humans were loss-aversive when involving in the gain/loss situations [34]. Although there was no direct evidence showing that experiencing negative gains would enhance performance optimality, it remained a possibility that the risk-seeking tendency under the "cliff" paradigm would diminish if the early/late responses were punished by penalty.

Why did some participants exhibit sub-optimality in the risk-before condition? We 
firstly argued that the asymmetry of the gain function (or risk-direction) was not the primary reason for inducing sub-optimality, as all participants achieved optimality in the risk-after condition. Previous studies [12 13] additionally proposed two types of distortions that may lead to sub-optimal performance with asymmetric gain functions: 1) distortion in numerical values, and 2) distortion in response variance. Distortion in numerical values was less likely occurring in our tasks because we provided both trial-by-trial points and block-averaged points as feedback which could potentially extenuate the value distortion 35, 36]. The implementation of adjustable risk-return control matching the response variance with the payoff variance could also reduce the chances of distortion in response variance. In concrete, a heuristic strategy was assumed to induce a risk-aversive tendency in our risk-before condition. Because higher points would be received as sooner the response was released after the target time, the "sub-optimal" participants might find it easy to earn points by relying on such a "simple reaction time" task. To sum up, at present, we have no clear explanation for the reason why participants could not achieve optimality only in the risk-before condition. Future studies are to clarify the strategy used by the "sub-optimal" participants.

Recent studies focused on modeling human decision-making under different risk-return configurations in either spatial tasks $4,5,24,26,28,37,37$ or temporal tasks [6, 12, 13, 16], and most studies only concerned with the motor-decision in one dimension. To step forward, our decision making-model incorporated the movement direction and timing response that controlled the variance and the mean of payoffs respectively. Such modeling might offer an application in daily life situations. For example, modeling bowling using balls with different sizes. Large balls are heavy and easy to slip out of the hand ( 0 points) but can potentially hit more pins (high-risk high-return), whereas small balls may hit fewer pins but can be controlled more precisely (low-risk low return). To gain more points, the player needs to consider the 
size of the ball before planning the hitting strategy. Here, our model could provide the prototype for the risk-return evaluation in such daily decisions.

\section{Author contributions}

We declare the following contributions made by authors.

Design: Qirui Yao, Yutaka Sakaguchi.

Data curation: Qirui Yao.

Data analysis: Qirui Yao.

Methodology: Qirui Yao.

Statistical modeling: Qirui Yao

Software \& Programming: Qirui Yao.

Supervision: Yutaka Sakaguchi.

Visualization: Qirui Yao

Writing - original draft: Qirui Yao, Yutaka Sakaguchi.

Writing - review \& editing: Yutaka Sakaguchi.

Funding acquisition: Yutaka Sakaguchi.

\section{Competing interests}

The authors declare no competing interests.

\section{Model availability}

The statistical models related to data analysis have been uploaded at https://github.co m/yty2020/stan-model. 


\section{Data Availability}

The data related to this study will be provided upon request.

\section{References}

1. Bernardo JM, Smith AF. Bayesian theory. vol. 405. and others, editor. John Wiley \& Sons; 2009.

2. Maloney LT, Mamassian P. Bayesian decision theory as a model of human visual perception: Testing Bayesian transfer. Visual Neuroscience. 2009;26(1):147-155.

3. Miyazaki M, Nozaki D, Nakajima Y. Testing Bayesian Models of Human Coincidence Timing. Journal of Neurophysiology. 2005;94(1):395-399.

4. Trommershäuser J, Landy MS, Maloney LT. Humans Rapidly Estimate Expected Gain in Movement Planning. Psychological Science. 2006;17(11):981-988.

5. Trommershäuser J, Maloney LT, Landy MS. Statistical decision theory and the selection of rapid, goal-directed movements. Journal of the Optical Society of America A. 2003;20(7):1419-1419.

6. Dean M, Wu SW, Maloney LT. Trading off speed and accuracy in rapid, goal-directed movements. Journal of Vision. 2007;7(5):10-10.

7. Bertucco M, Bhanpuri NH, Sanger TD. Perceived Cost and Intrinsic Motor Variability Modulate the Speed-Accuracy Trade-Off. PLOS ONE. 2015;10(10):e0139988-e0139988.

8. Nagengast AJ, Braun DA, Wolpert DM. Risk sensitivity in a motor task with speed-accuracy trade-off. Journal of Neurophysiology. 2011;105(6):2668-2674. 
9. Fishbach A, Mussa-Ivaldi FA. Seeing versus Believing: Conflicting Immediate and Predicted Feedback Lead to Suboptimal Motor Performance. Journal of Neuroscience. 2008;28(52):14140-14146.

10. Liesker H, Brenner E, Smeets JBJ. Combining eye and hand in search is suboptimal. Experimental Brain Research. 2009;197(4):395-401.

11. Veilleux LN, Proteau L. Suboptimal online control of aiming movements in virtual contexts. Experimental Brain Research. 2011;208(3):345-358.

12. Ota K, Shinya M, Kudo K. Motor planning under temporal uncertainty is suboptimal when the gain function is asymmetric. Frontiers in Computational Neuroscience. 2015;9(9):88-88.

13. Ota K, Shinya M, Kudo K. Sub-optimality in motor planning is retained throughout 9 days practice of 2250 trials. Scientific Reports. 2016;6(1):37181-37181.

14. Ota K, Shinya M, Maloney LT, Kudo K. Sub-optimality in motor planning is not improved by explicit observation of motor uncertainty. Scientific Reports. 2019;9(1):1-11.

15. Wu SW, Martello MFD, Maloney LT. Sub-Optimal Allocation of Time in Sequential Movements. PLoS ONE. 2009;4(12):e8228-e8228.

16. Hudson TE, Maloney LT, Landy MS. Optimal Compensation for Temporal Uncertainty in Movement Planning. PLoS Computational Biology. 2008;4(7):e1000130-e1000130.

17. Nagengast AJ, Braun DA, Wolpert DM. Risk-sensitivity and the mean-variance trade-off: decision making in sensorimotor control. Proceedings of the Royal Society B: Biological Sciences. 1716;278:2325-2332. 
18. Inquisit 5 [Computer software]; 2016. Available from: https://www.millisecond.com.

19. MATLAB and Statistics Toolbox Release. The MathWorks, Inc; 2012.

20. Mosteller F, Tukey JW. Data analysis and regression: a second course in statistics. Behavioral Science: Quantitative Methods. 1977;.

21. RStan: The R interface to Stan. Stan Development Team. 2016;.

22. Bates D, Mächler M, Bolker B, Walker S. Fitting Linear Mixed-Effects Models Usinglme4. Journal of Statistical Software. 2015;67(1):1-48.

23. Trommershäuser J, Gepshtein S, Maloney LT, Landy MS, Banks MS. Optimal compensation for changes in task-relevant movement variability. Journal of Neuroscience. 2005;25(31):7169-7178.

24. Maloney L, Landy M, Trommershäuser J. Statistical decision theory and trade-offs in the control of motor response. Spatial Vision. 2003;16(3):255-275.

25. Magezi DA. Linear mixed-effects models for within-participant psychology experiments: an introductory tutorial and free, graphical user interface (LMMgui). Frontiers in Psychology. 2015;6(2).

26. O'Brien MK, Ahmed AA. Does risk-sensitivity transfer across movements? Journal of neurophysiology. 2013;109(7):1866-1875.

27. Wu SW, Trommershäuser J, Maloney LT, Landy MS. Limits to human movement planning in tasks with asymmetric gain landscapes. Journal of Vision. $2006 ; 6(1): 5-5$.

28. Wu SW, Delgado MR, Maloney LT. Economic decision-making compared with an equivalent motor task. Proceedings of the National Academy of Sciences. 2009;106(15):6088-6093. 
29. Hudson TE, Tassinari H, Landy MS. Compensation for Changing Motor Uncertainty. PLoS Computational Biology. 2010;6(11):e1000982-e1000982.

30. Zhang H, Daw ND, Maloney LT. Testing Whether Humans Have an Accurate Model of Their Own Motor Uncertainty in a Speeded Reaching Task. PLoS Computational Biology. 2013;9(5):e1003080-e1003080.

31. Lewis PA, Miall RC. A right hemispheric prefrontal system for cognitive time measurement. Behavioural Processes. 2006;71(2-3):226-234.

32. Rakitin BC, Gibbon J, Penney TB, Malapani C, Hinton SC, Meck WH. Scalar expectancy theory and peak-interval timing in humans. Journal of Experimental Psychology: Animal Behavior Processes. 1998;24(1):15-33.

33. Acerbi L, Wolpert DM, Vijayakumar S. Internal Representations of Temporal Statistics and Feedback Calibrate Motor-Sensory Interval Timing. PLoS Computational Biology. 2012;8(11):e1002771-e1002771.

34. Tom SM, Fox CR, Trepel C, Poldrack RA. The Neural Basis of Loss Aversion in Decision-Making Under Risk. Science. 2007;315(5811):515-518.

35. Neyedli HF, Welsh TN. Participants are better at maximizing expected gain in a manual aiming task with rapidly changing probabilities rather than rapidly changing payoffs. Movement, and Sport (SCAPPS refereed abstracts repository). 2012;44:47-47.

36. Neyedli HF, Welsh TN. Optimal weighting of costs and probabilities in a risky motor decision-making task requires experience. Journal of experimental psychology: human perception and performance. 2013;39(3):638.

37. Ota K, Tanae M, Ishii K, Takiyama K. Optimizing motor decision-making through competition with opponents. Scientific Reports. 2020;10(1):1-14. 


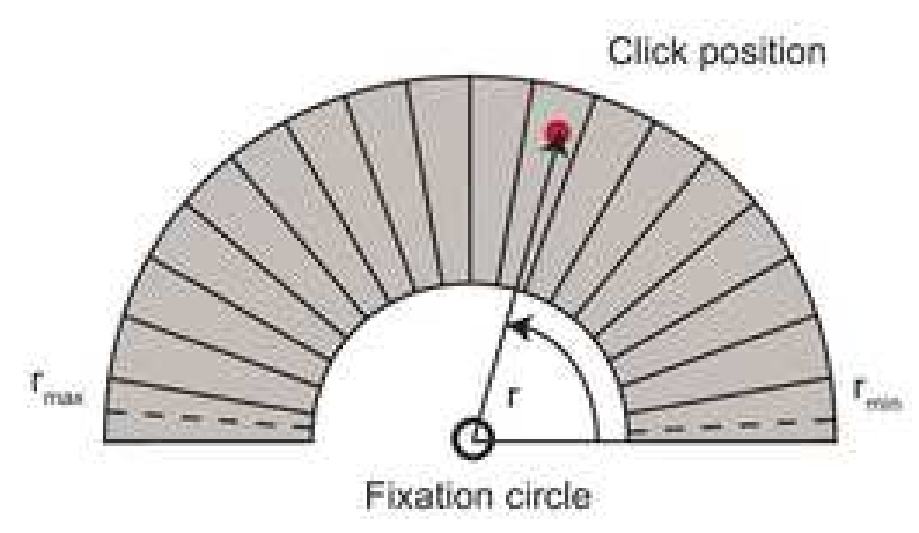

1st tone

2nd tone

3rd tone

Fixation circle

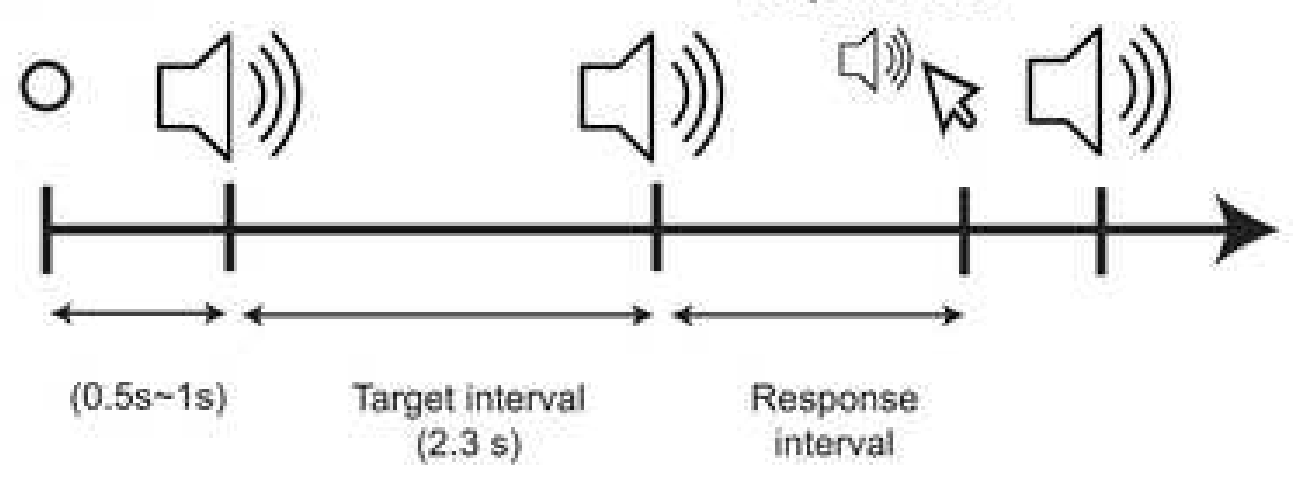




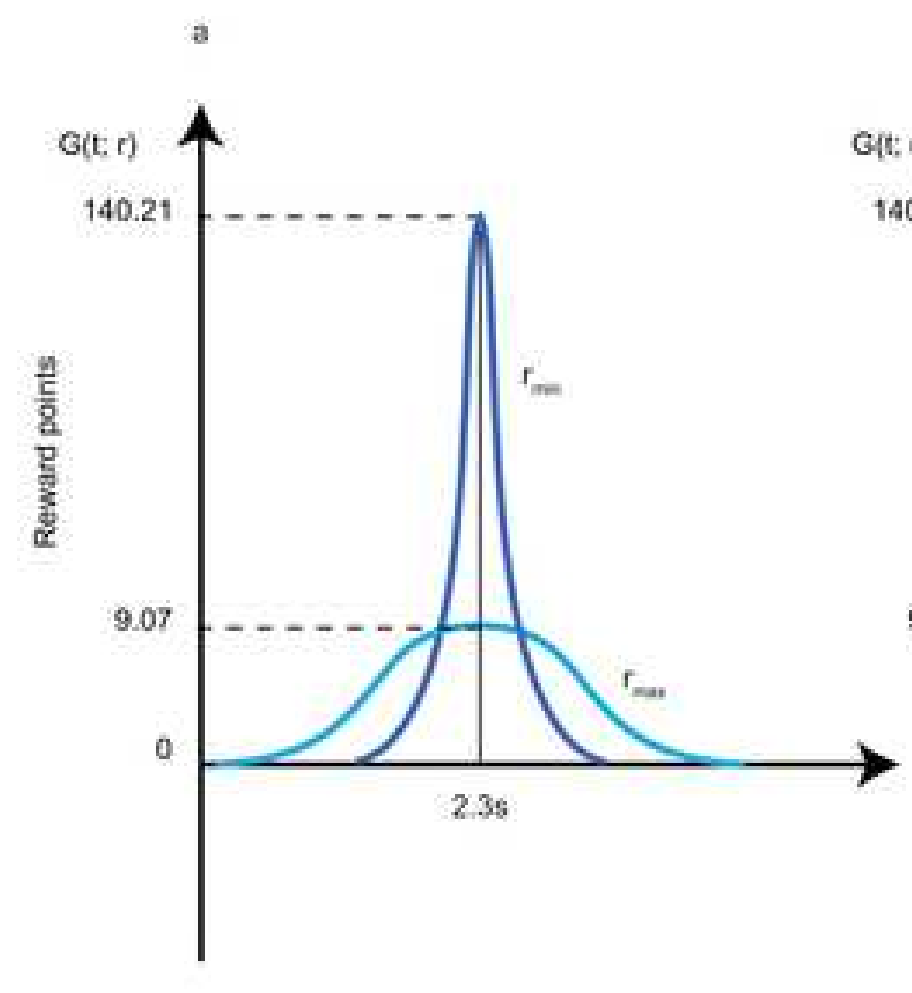

b

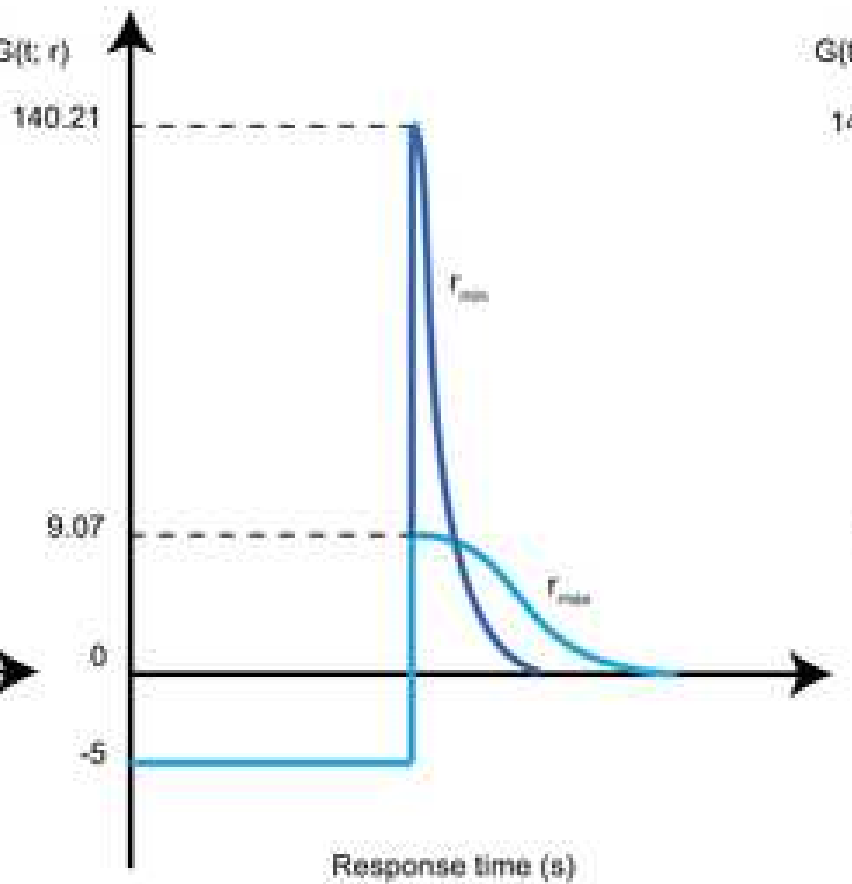

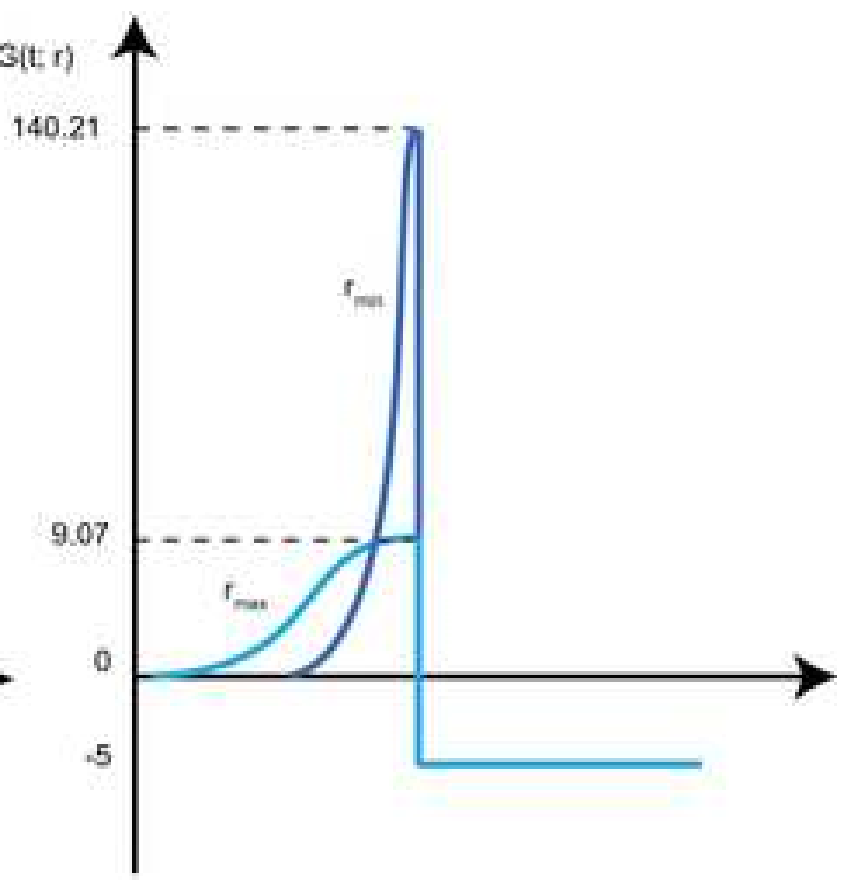


fig3

Click here to access/download;Figure;fig3.tif $\underline{\underline{ }}$
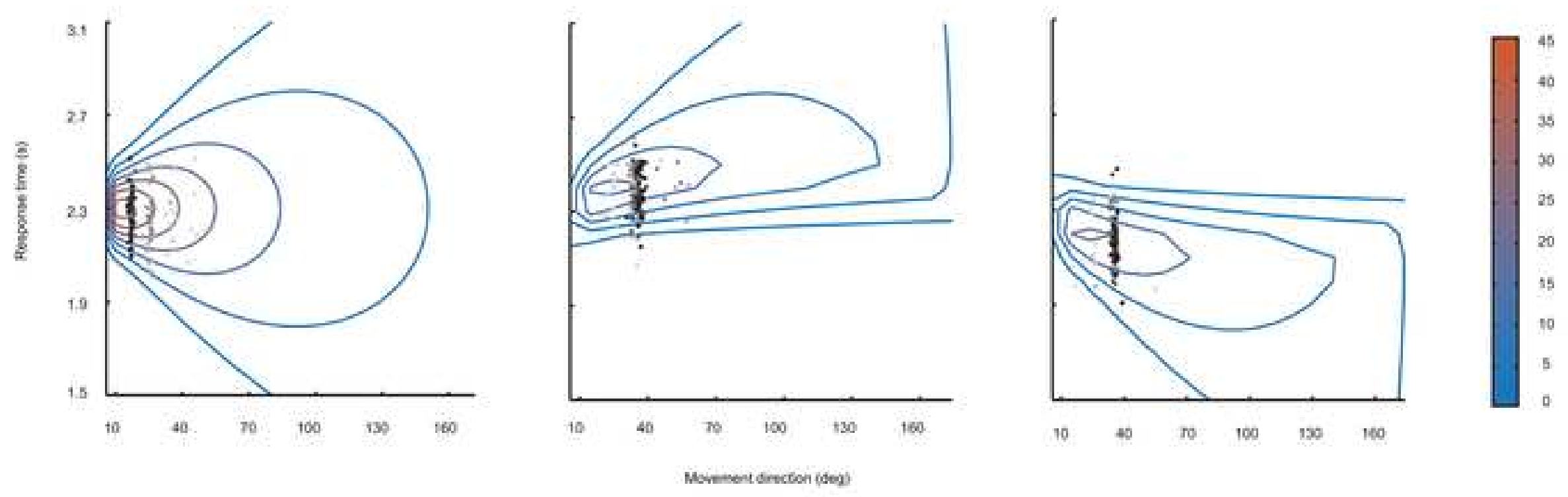


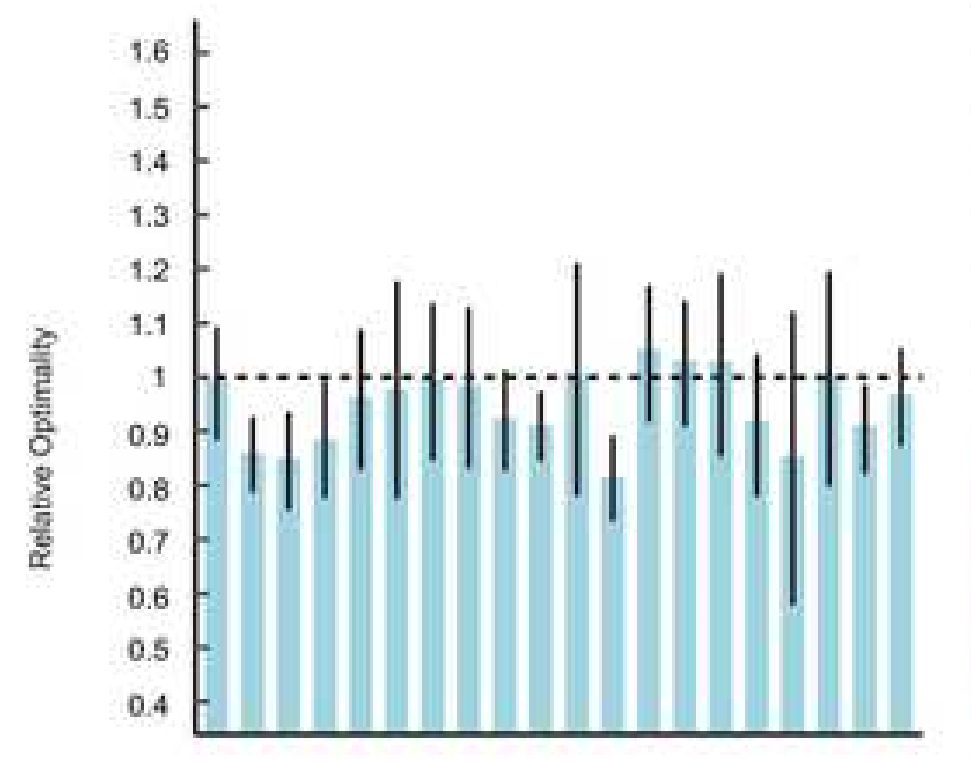

b

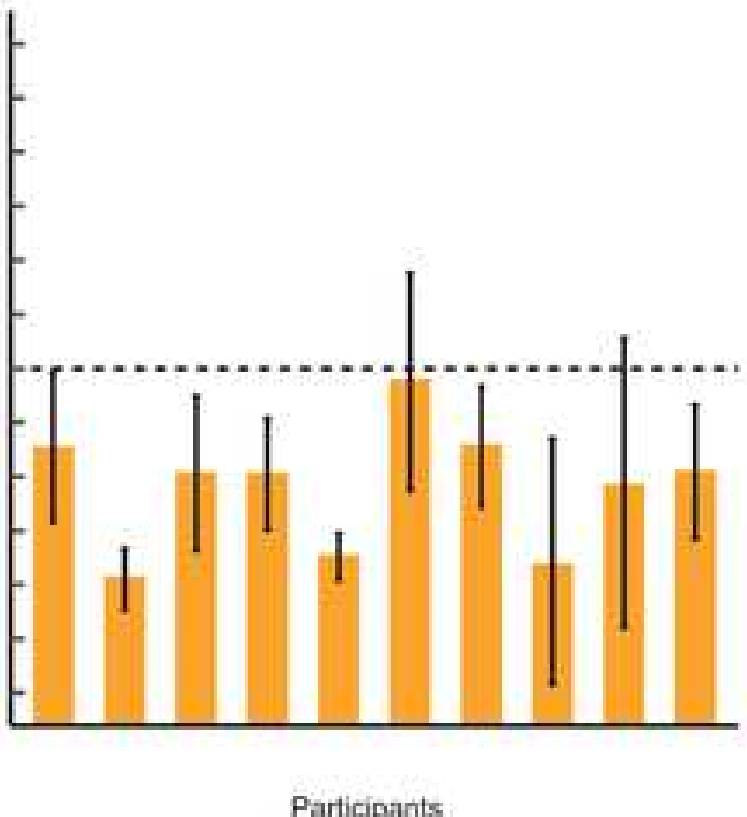

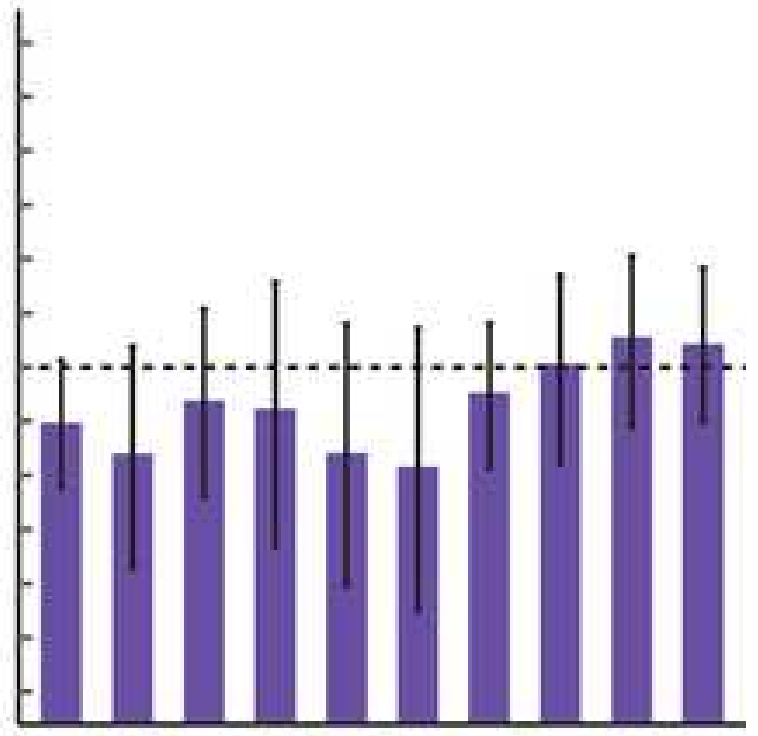




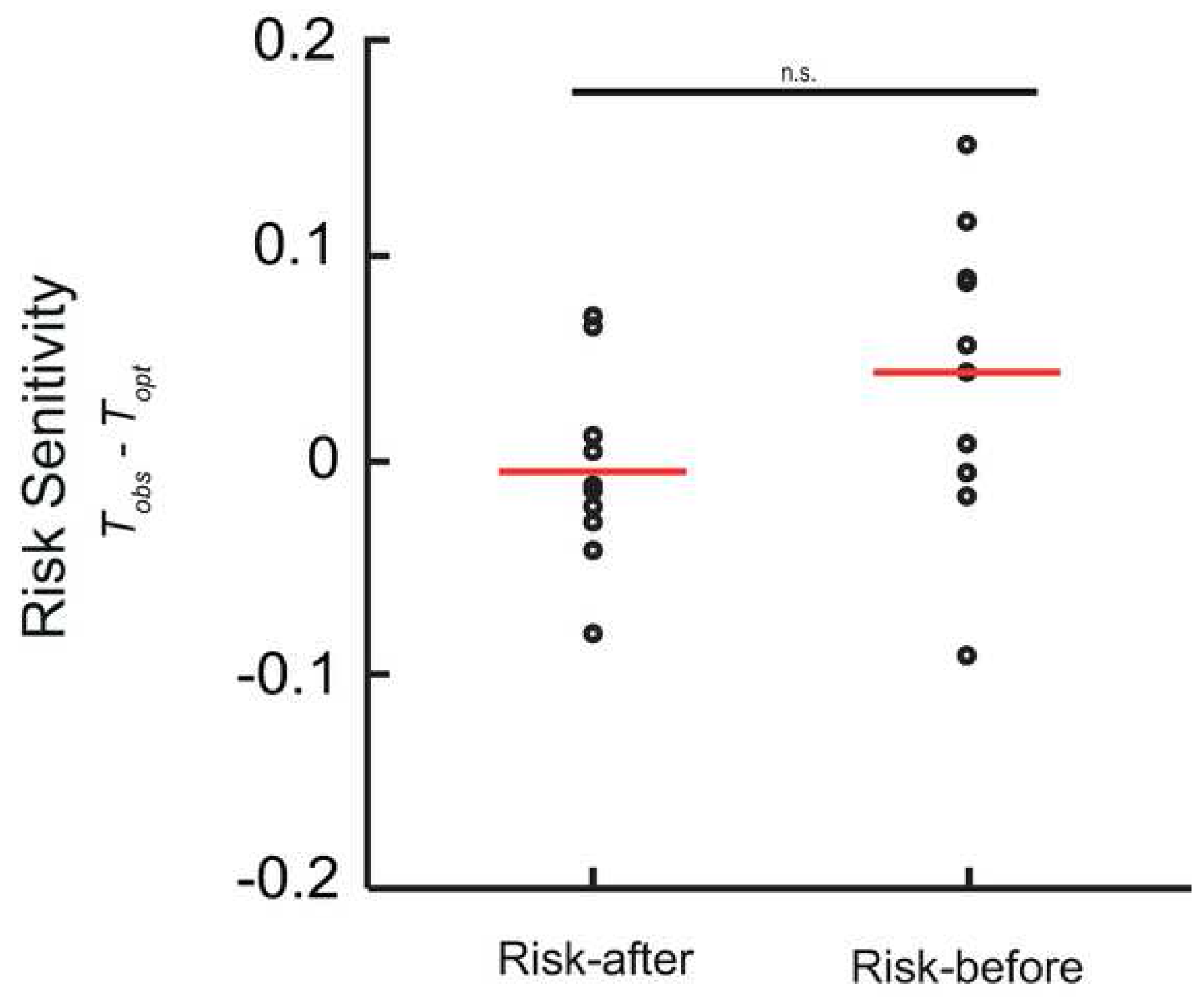

Risk Conditions 


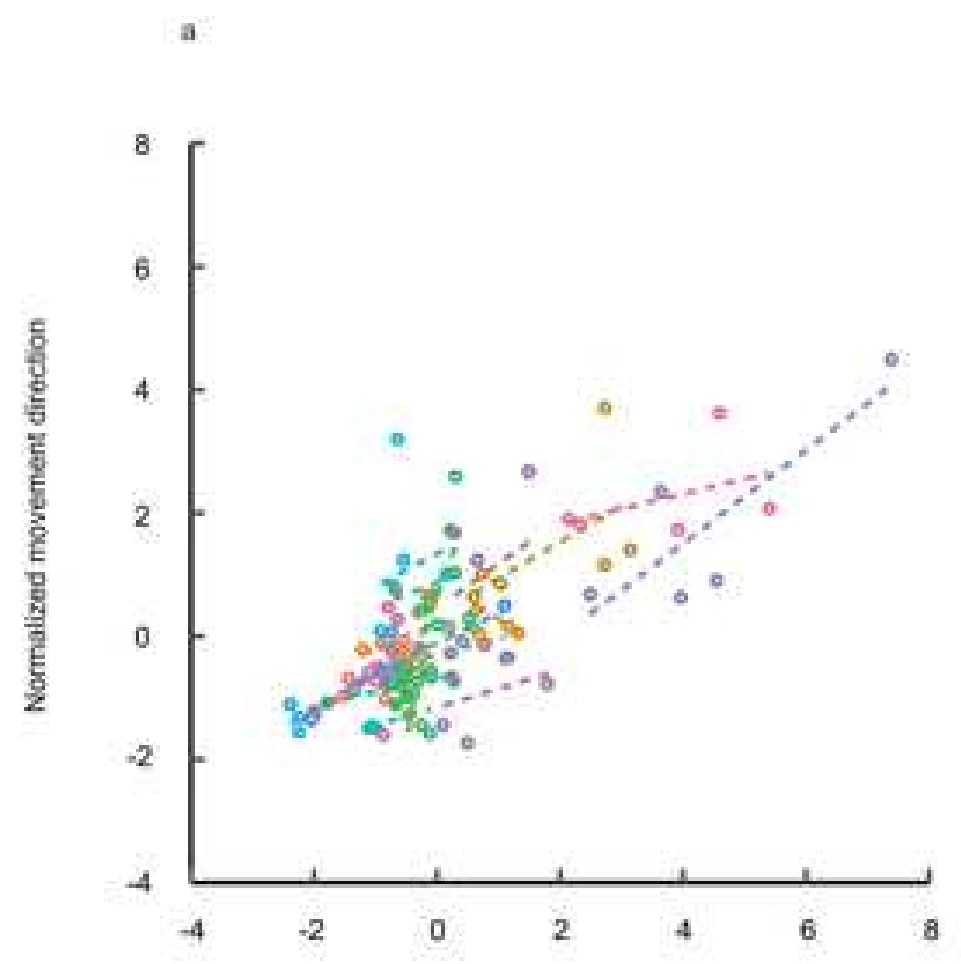

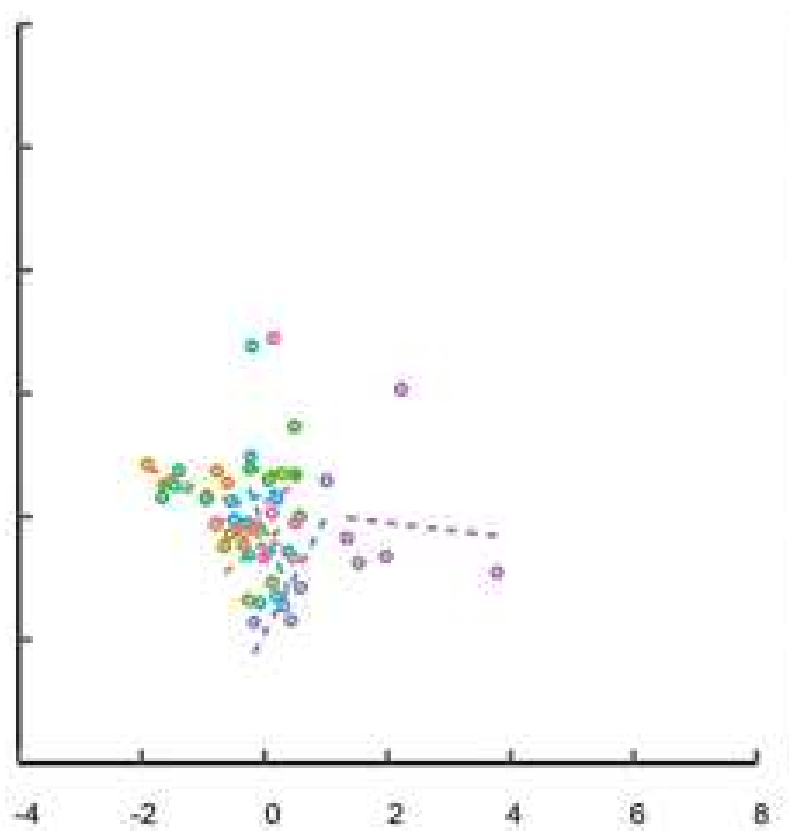

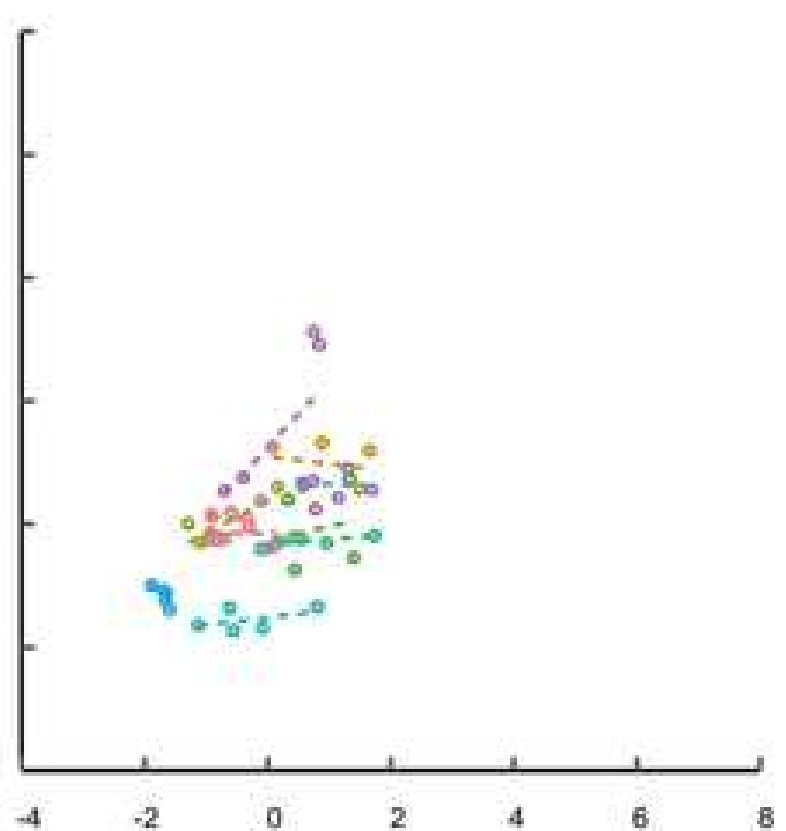

Noramzalized timing variance 
Click here to access/download Supporting Information S1 Fig.tif 
Click here to access/download Supporting Information S2 Fig.tif 
Click here to access/download Supporting Information S3 Fig.tif 
Click here to access/download Supporting Information S4 Fig.tif 
Click here to access/download Supporting Information Supplementary_Material.docx 